\title{
ON THE KÜNNETH FORMULA FOR INTERSECTION COHOMOLOGY
}

\author{
DANIEL C. COHEN, MARK GORESKY, AND LIZHEN JI
}

ABSTRACT. We find the natural perversity functions for which intersection cohomology satisfies the Künneth formula.

\section{INTRODUCTION}

It was first shown by J. Cheeger [Ch] that the Künneth formula holds for the "middle" intersection cohomology, i.e.,

$$
I^{\bar{m}} H^{i}(X \times Y ; \mathbb{R}) \cong \bigoplus_{a+b=i} I^{\bar{m}} H^{a}(X ; \mathbb{R}) \otimes I^{\bar{m}} H^{b}(Y ; \mathbb{R})
$$

where $X$ and $Y$ are compact pseudomanifolds with even codimension strata. It has been observed that for any perversity $\bar{p}$ and for any compact pseudomanifold $X$, there is a short exact sequence for intersection cohomology with integer coefficients,

$0 \rightarrow \bigoplus_{a+b=i} I^{\bar{p}} H^{a}(X) \otimes H^{b}(M) \rightarrow I^{\bar{p}} H^{i}(X \times M) \rightarrow \bigoplus_{a+b=i-1} \operatorname{Tor}\left(I^{\bar{p}} H^{a}(X), H^{b}(M)\right) \rightarrow 0$

provided $M$ is a compact manifold (compare [Ki]). On the other hand, for most perversities $\bar{p}$, the intersection cohomology groups $I^{\bar{p}} H^{i}(X \times Y)$ do not satisfy the Künneth formula (see counterexamples in $\S 5$ ). In this note we find the natural perversity functions $\bar{p}$ for which $I^{\bar{p}} H^{*}$ satisfies the Künneth formula.

Fix a coefficient ring $R$ which is a principal ideal domain and suppose $X$ and $Y$ are compact pseudomanifolds. Let $\bar{p}$ be a perversity. Recall ([GS]) that the pseudomanifold $X$ is locally $\bar{p}$-torsion free (over $R$ ) if, for each stratum $S$ of $X$, the link $L$ of $S$ satisfies

$$
I^{\bar{p}} T^{c-p(c)-2}(L ; R)=0 \quad \text { where } c=\operatorname{codim}(S),
$$

and $I^{\bar{p}} T^{i}(L ; R)$ denotes the torsion subgroup of $I^{\bar{p}} H^{i}(L ; R)$.

We consider two cases:

Case (A). Suppose that the perversity $\bar{p}$ satisfies $p(a)+p(b) \leq p(a+b) \leq$ $p(a)+p(b)+1$ for all $a$ and $b$.

Case (B). Suppose that the perversity $\bar{p}$ satisfies $p(a)+p(b) \leq p(a+b) \leq$ $p(a)+p(b)+2$ for all $a$ and $b$, and assume that either $X$ or $Y$ is locally $\bar{p}$-torsion free (over $R$ ).

Received by the editors December 27, 1989.

1991 Mathematics Subject Classification. Primary 57N80; Secondary 55N30, 55N45.

The second author was partially supported by NSF grant \# DMS 88022638 . 
Theorem 1. In either case (A) or (B) above, there is a split short exact sequence for intersection cohomology with perversity $\bar{p}$ and with coefficients in $R$,

$$
0 \rightarrow \bigoplus_{a+b=i} I H^{a}(X) \otimes I H^{b}(Y) \rightarrow I H^{i}(X \times Y) \rightarrow \bigoplus_{a+b=i-1} \operatorname{Tor}^{R}\left(I H^{a}(X), I H^{b}(Y)\right) \rightarrow 0 .
$$

Remarks. 1. If $R$ is a field then all pseudomanifolds are locally $\bar{p}$-torsion free over $R$, and the theorem says that the intersection cohomology of $X \times Y$ is the tensor product of the intersection cohomology of $X$ with that of $Y$, provided that the perversity satisfies the inequalities in condition (B) above.

2. If $Y$ is a manifold then it is locally $\bar{p}$-torsion free for any perversity $\bar{p}$, and its intersection cohomology is just the usual cohomology, so we recover the case studied in [Ki].

3. All pseudomanifolds are locally $\bar{t}$-torsion free, where $\bar{t}$ denotes the "top" perversity, and Theorem 1 is the Künneth formula in ordinary homology.

4. The condition on the perversity $\bar{p}$ means that the graph of the perversity function does not deviate far from some straight line through the origin. Perversities with this property also arise in connection with $L^{q}$-cohomology [BGM].

5. More generally, one may start with a local system $\mathscr{L}_{X}$ of $R$-modules on the nonsingular part of $X$, and a local system $\mathscr{L}_{Y}$ of $R$-modules on the nonsingular part of $Y$. Then the same result holds if we replace $I H^{*}(X)$ by $I H^{*}\left(X ; \mathscr{L}_{X}\right), I H^{*}(Y)$ by $I H^{*}\left(Y ; \mathscr{L}_{Y}\right)$, and $I H^{*}(X \times Y)$ by $I H^{*}\left(X \times Y ; \mathscr{L}_{X} \otimes \mathscr{L}_{Y}\right)$. Note however, that the definition of locally $\bar{p}$-torsion free involves the group $I^{\bar{p}} H^{c-p(c)-2}\left(L ;\left.\mathscr{L}_{X}\right|_{L}\right)$, which may vary as the local system $\mathscr{L}_{X}$ varies. Since the proof is the same, we will omit further mention of local systems.

6. If $X$ or $Y$ is not compact, the same result holds provided we use compactly supported intersection cohomology throughout.

We would like to thank David Massey and David Yavin for useful conversations.

\section{THE KÜNNETH THEOREM IN SHEAF COHOMOLOGY}

Suppose $X$ and $Y$ are pseudomanifolds, but are not necessarliy compact. Let $\mathbf{I}^{\overline{\mathbf{p}}} \mathbf{C}^{\bullet}(X ; R)$ denote the complex of sheaves of intersection cochains on $X$, with coefficients in the ring $R$ [GM2]. Let $\pi_{1}$ and $\pi_{2}$ denote the projections of $X \times Y$ to $X$ and $Y$ respectively. It follows from sheaf theory [Bo, V, Theorem 10.19 , p. 170; Iv, VII, 2.7 , p. 323] that the compactly supported cohomology of the complex of sheaves

$$
\mathbf{S}^{\bullet}=\pi_{1}^{*}\left(\mathbf{I}^{\overline{\mathbf{p}}} \mathbf{C}^{\bullet}(X)\right) \otimes \pi_{2}^{*}\left(\mathbf{I}^{\overline{\mathbf{p}}} \mathbf{C}^{\bullet}(Y)\right)
$$

satisfies the Künneth formula, i.e., it fits into the short exact sequence

$$
\begin{aligned}
0 \rightarrow \bigoplus_{a+b=i} I H_{c}^{a}(X) \otimes I H_{c}^{b}(Y) \rightarrow H_{c}^{i}(X \times & \left.; \mathbf{S}^{\bullet}\right) \\
& \rightarrow \bigoplus_{a+b=i-1} \operatorname{Tor}^{R}\left(I H_{c}^{a}(X), I H_{c}^{b}(Y)\right) \rightarrow 0
\end{aligned}
$$

where $I H_{c}$ denotes intersection cohomology with perversity $\bar{p}$, compact supports, and coefficients in $R$. Therefore it suffices to prove the following result: 
Proposition 2. In either case (A) or (B) above, the multiplication map which is defined on the nonsingular part $X^{\circ} \times Y^{\circ}$ of $X \times Y$,

$$
\pi_{1}^{*}\left(\mathbf{R}_{X^{\circ}}\right) \otimes \pi_{2}^{*}\left(\mathbf{R}_{Y^{\circ}}\right) \rightarrow \mathbf{R}_{X^{\circ} \times Y^{\circ}},
$$

extends to a unique quasi-isomorphism,

$$
\pi_{1}^{*}\left(\mathbf{I}^{\overline{\mathbf{p}}} \mathbf{C}^{\bullet}(X ; R)\right) \otimes \pi_{2}^{*}\left(\mathbf{I}^{\overline{\mathbf{p}}} \mathbf{C}^{\bullet}(Y ; R)\right) \cong \mathbf{I}^{\overline{\mathbf{p}}} \mathbf{C}^{\bullet}(X \times Y ; R) .
$$

\section{INTERSECTION COHOMOLOGY OF A JOIN}

In this section we assume that $X$ and $Y$ are compact. As a corollary to Theorem 1, we compute $I^{\bar{p}} H^{*}(X * Y)$, where $X * Y$ denotes the join of $X$ and $Y$.

Proposition 3. Let $X$ and $Y$ be pseudomanifolds of dimensions $m-1$ and $n-1$. In either case (A) or (B) above, we have $I^{\bar{p}} H^{i}(X * Y)=$

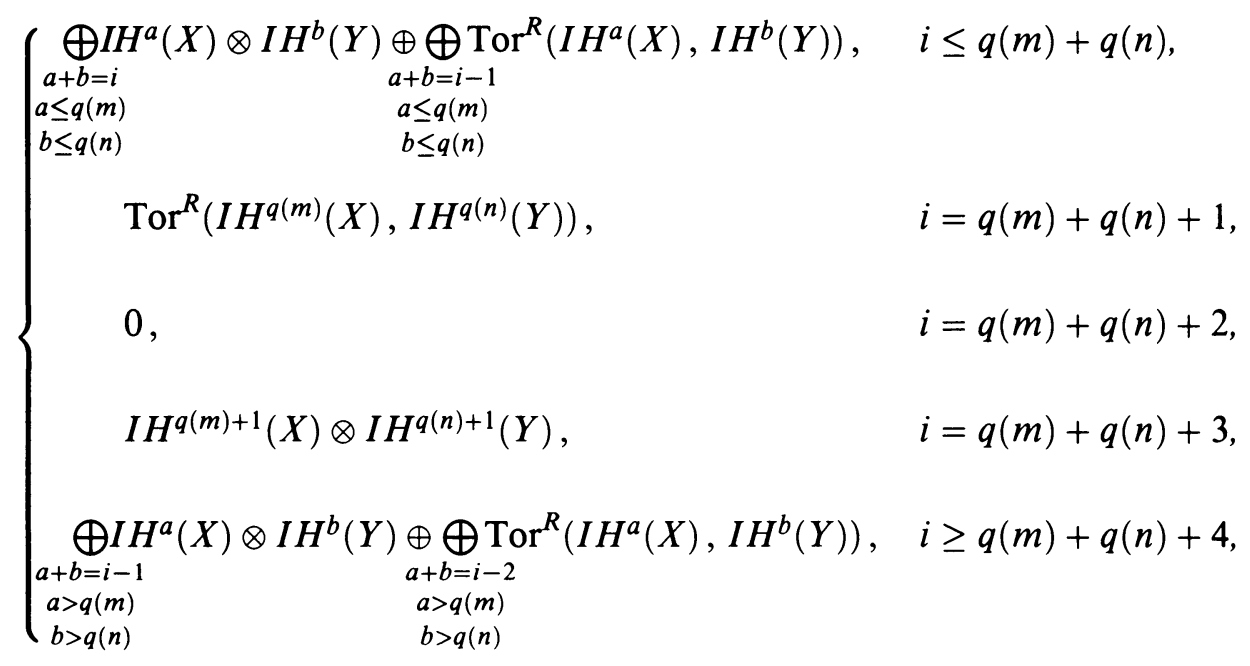

where $q(m)=m-p(m)-2$ and $q(n)=n-p(n)-2$.

Notice that the group $\operatorname{Tor}^{R}\left(I H^{q(m)}(X), I H^{q(n)}(Y)\right)$ vanishes in case (B).

Proof. Cover the join $X * Y$ by two subsets $U \cong X \times c Y$ and $V \cong c X \times Y$, whose intersection is $U \cap V \cong X \times Y \times\left[\frac{1}{2}-\varepsilon, \frac{1}{2}+\varepsilon\right]$. We shall compute $I H^{*}(X * Y)$ using Mayer-Vietoris and the Künneth formula for each of the three subsets. Consider the Mayer-Vietoris sequence in intersection cohomology

$\cdots \stackrel{\Delta_{i-1}}{\longrightarrow} I H^{i}(X * Y) \stackrel{\Phi_{i}}{\longrightarrow} I H^{i}(X \times c Y) \oplus I H^{i}(c X \times Y) \stackrel{\Psi_{i}}{\longrightarrow} I H^{i}(X \times Y) \stackrel{\Delta_{i}}{\longrightarrow} \cdots$.

By Theorem 1, the Künneth formula holds for the intersection cohomology groups of $X \times Y, X \times c Y$, and $c X \times Y$. So we have a diagram with split short exact rows 


$$
\begin{aligned}
& 0 \rightarrow \bigoplus_{a+b=i} I H^{a}(X) \otimes I H^{b}(Y) \quad \rightarrow \quad I H^{i}(X \times Y) \quad \rightarrow \bigoplus_{a+b=i-1} \operatorname{Tor}^{R}\left(I H^{a}(X), I H^{b}(Y)\right) \rightarrow 0
\end{aligned}
$$

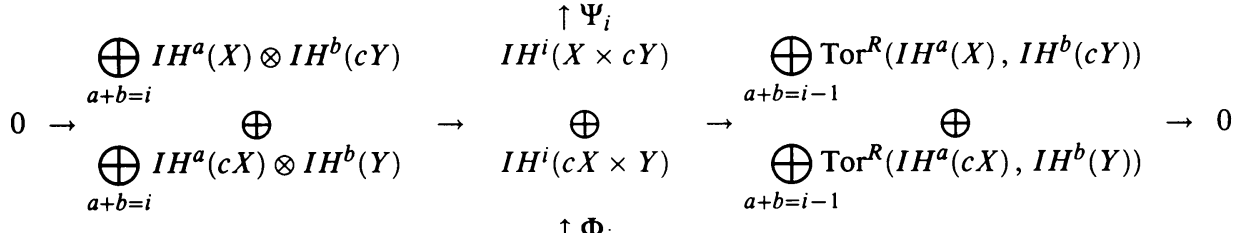

$$
\begin{aligned}
& \begin{array}{c}
\uparrow \Phi_{i} \\
I H^{i}(X * Y)
\end{array}
\end{aligned}
$$

Using the computation of the intersection cohomology of a cone [GM1], we observe that, for instance

$$
\bigoplus_{a+b=i} I H^{a}(X) \otimes I H^{b}(c Y)=\bigoplus_{\substack{a+b=i \\ b \leq q(n)}} I H^{a}(X) \otimes I H^{b}(Y)
$$

is a subgroup of $\bigoplus_{a+b=i} I H^{a}(X) \otimes I H^{b}(Y)$. Thus there are homomorphisms $\alpha_{i}$ and $\beta_{i}$ so that the following diagram commutes:

$$
\begin{aligned}
& 0 \rightarrow \bigoplus_{a+b=i} I H^{a}(X) \otimes I H^{b}(Y) \rightarrow I H^{i}(X \times Y) \rightarrow \bigoplus_{a+b=i-1} \operatorname{Tor}^{R}\left(I H^{a}(X), I H^{b}(Y)\right) \rightarrow 0 \\
& \begin{array}{ccc}
\uparrow \alpha_{i} & \uparrow \Psi_{i} & \uparrow \beta_{i} \\
\underset{\substack{a+b=i \\
b \leq q(n)}}{ } I H^{a}(X) \otimes I H^{b}(Y) & I H^{i}(X \times c Y) & \bigoplus_{\substack{a+b=i-1 \\
b \leq q(n)}} \operatorname{Tor}^{R}\left(I H^{a}(X), I H^{b}(Y)\right)
\end{array} \\
& 0 \rightarrow \bigoplus_{\substack{a+b=i \\
a \leq q(m)}} I H^{a}(X) \otimes I H^{b}(Y) \quad \rightarrow \quad \underset{I H^{i}(c X \times Y)}{\bigoplus} \rightarrow \underset{\substack{a+b=i-1 \\
a \leq q(m)}}{\bigoplus \operatorname{Tor}^{R}\left(I H^{a}(X), I H^{b}(Y)\right)} \rightarrow 0 \\
& \begin{array}{c}
\uparrow \Phi_{i} \\
I H^{i}(X * Y)
\end{array}
\end{aligned}
$$

Check that:

$$
\begin{aligned}
\operatorname{ker}\left(\alpha_{i}\right)= \begin{cases}\bigoplus_{\substack{a+b=i \\
a \leq q(m) \\
b \leq q(n)}} I H^{a}(X) \otimes I H^{b}(Y), & i \leq q(m)+q(n), \\
0, & i>q(m)+q(n),\end{cases} \\
\operatorname{coker}\left(\alpha_{i}\right)= \begin{cases}0, & i \leq q(m)+q(n)+1, \\
\bigoplus_{a+b=i} I H^{a}(X) \otimes I H^{b}(Y), & i>q(m)+q(n)+1, \\
a>q(m) \\
b>q(n)\end{cases} \\
\operatorname{ker}\left(\beta_{i}\right)= \begin{cases}\bigoplus \operatorname{Tor}^{R}\left(I H^{a}(X), I H^{b}(Y)\right), & i \leq q(m)+q(n)+1, \\
a+b=i-1 \\
a \leq q(m) \\
b \leq q(n) \\
0,\end{cases}
\end{aligned}
$$




$$
\operatorname{coker}\left(\beta_{i}\right)=\left\{\begin{array}{cl}
0, & i \leq q(m)+q(n)+2 \\
\bigoplus_{\substack{a+b=i-1 \\
a>q(m) \\
b>q(n)}} \operatorname{Tor}^{R}\left(I H^{a}(X), I H^{b}(Y)\right), & i>q(m)+q(n)+2
\end{array}\right.
$$

By splicing together short exact sequences involving the kernels and cokernels of the maps $\alpha_{i}$ and $\beta_{i}$, we obtain the following diagram with everywhere exact columns and split exact rows:

$$
\begin{aligned}
& 0 \rightarrow \bigoplus_{a+b=i} I H^{a}(X) \otimes I H^{b}(Y) \rightarrow I H^{i}(X \times Y) \rightarrow \bigoplus_{a+b=i-1} \operatorname{Tor}^{R}\left(I H^{a}(X), I H^{b}(Y)\right) \rightarrow 0 \\
& \begin{array}{ccc}
\uparrow \alpha_{i} & \uparrow \Psi_{i} & \uparrow \beta_{i} \\
\bigoplus_{a+b=i} I H^{a}(X) \otimes I H^{b}(Y) & I H^{i}(X \times c Y) & \bigoplus_{\substack{a+b=i-1 \\
b \leq q(n)}} \operatorname{Tor}^{R}\left(I H^{a}(X), I H^{b}(Y)\right)
\end{array}
\end{aligned}
$$

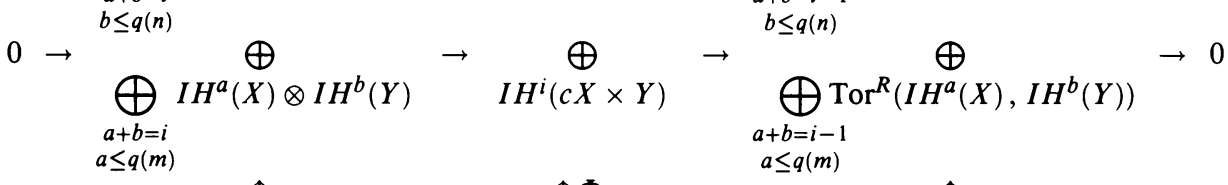

$$
\begin{aligned}
& \begin{array}{ccc}
\uparrow & \uparrow \Phi_{i} & \uparrow \\
\operatorname{ker}\left(\alpha_{i}\right) \oplus \operatorname{coker}\left(\alpha_{i-1}\right) & I H^{i}(X * Y) & \operatorname{ker}\left(\beta_{i}\right) \oplus \operatorname{coker}\left(\beta_{i-1}\right)
\end{array}
\end{aligned}
$$

For $i \leq q(m)+q(n)+2$, the maps $\alpha_{i-1}$ and $\beta_{i-1}$ are surjective by the computation of the intersection cohomology of a cone. It follows that $\Psi_{i-1}$ is also surjective, hence $\Phi_{i}$ is injective. Similarly, since $\alpha_{i}$ and $\beta_{i}$ are injective for $i \geq q(m)+q(n)+3, \Phi_{i}$ is the zero map in these dimensions.

Using these facts, it is an exercise in homological algebra to verify that

$$
0 \rightarrow \operatorname{ker}\left(\alpha_{i}\right) \rightarrow I H^{i}(X * Y) \rightarrow \operatorname{ker}\left(\beta_{i}\right) \rightarrow 0
$$

is split exact for $i \leq q(m)+q(n)+2$, and that

$$
0 \rightarrow \operatorname{coker}\left(\alpha_{i-1}\right) \rightarrow I H^{i}(X * Y) \rightarrow \operatorname{coker}\left(\beta_{i-1}\right) \rightarrow 0
$$

is split exact for $i \geq q(m)+q(n)+3$. Direct computations involving these sequences finish the proof of the proposition.

\section{Proof of Proposition 2}

We will use induction on $k=\operatorname{dim}(X)+\operatorname{dim}(Y)$ with the case $k=0$ trivial. We shall refer to Theorem $1_{k}$ and Proposition $2_{k}$ when the additional hypothesis $\operatorname{dim}(X)+\operatorname{dim}(Y) \leq k$ is satisfied. Similarly, we refer to Proposition $3_{k}$ when the additional hypothesis $m+n-1 \leq k$ is satisfied. Then Proposition $2_{k-1} \Rightarrow$ Theorem $1_{k-1} \Rightarrow$ Proposition $3_{k-1}$ and we now show that Proposition $3_{k-1} \Rightarrow$ Proposition $22_{k}$.

The multiplication map $\pi_{1}^{*}\left(\mathbf{R}_{X^{\circ}}\right) \otimes \pi_{2}^{*}\left(\mathbf{R}_{Y^{\circ}}\right) \rightarrow \mathbf{R}_{X^{\circ} \times Y^{\circ}}$ is a quasi-isomorphism (in fact it is an isomorphism). Let us assume (by induction on $t$ ) that this extends uniquely to a quasi-isomorphism

$$
\left.\pi_{1}^{*}\left(\mathbf{I}^{\overline{\mathbf{p}}} \mathbf{C}^{\bullet}(X ; R)\right) \otimes \pi_{2}^{*}\left(\mathbf{I}^{\overline{\mathbf{p}}} \mathbf{C}^{\bullet}(Y ; R)\right)\right|_{(X \times Y-V)} \cong \mathbf{I}^{\overline{\mathbf{p}}} \mathbf{C}^{\bullet}(X \times Y-V ; R)
$$

over the complement of the union $V$ of all strata with codimension greater than $t-1$. We must show that the quasi-isomorphism extends over the complement of the set of strata with codimension greater than $t$. Let $(x, y) \in X \times Y$ be a point in the complement of the union of all strata of codimension greater than $t$. By [GM2] it suffices to check that $\mathbf{I C}^{\bullet}(X) \otimes \mathbf{I C}^{\bullet}(Y)$ and $\mathbf{I C}^{\bullet}(X \times Y)$ 
have the same stalk cohomology at $p=(x, y)$. Let $U_{x}$ and $U_{y}$ denote basic open neighborhoods in $X$ and $Y$ respectively, of the points $x$ and $y$. The local cohomology at $p$ of the sheaf $\operatorname{IC}^{\bullet}(X) \otimes \mathrm{IC}^{\bullet}(Y)$ is given by the Künneth formula discussed in $\S 2$, namely:

$$
H_{p}^{i}\left(\mathbf{I C}^{\bullet}(X) \otimes \mathbf{I C}^{\bullet}(Y)\right) \cong \bigoplus_{a+b=i} I H^{a}\left(U_{x}\right) \otimes I H^{b}\left(U_{y}\right) \oplus \bigoplus_{a+b=i-1} \operatorname{Tor}^{R}\left(I H^{a}\left(U_{x}\right), I H^{b}\left(U_{y}\right)\right) \text {. }
$$

There are homeomorphisms $U_{x} \cong E \times c L_{x}$ and $U_{y} \cong E \times c L_{y}$, where $L_{x}$ and $L_{y}$ are the links of $x$ and $y$ in $X$ and $Y$ respectively, and $E$ is a disk of suitable dimension in each case. Since the Künneth formula in intersection cohomology holds for a product with a disk [GM2], we have

$$
\begin{aligned}
H_{p}^{i}\left(\mathbf{I C}^{\bullet}(X) \otimes \mathbf{I C}^{\bullet}(Y)\right) \cong \\
\qquad \bigoplus_{a+b=i} I H^{a}\left(c L_{x}\right) \otimes I H^{b}\left(c L_{y}\right) \oplus \bigoplus_{a+b=i-1} \operatorname{Tor}^{R}\left(I H^{a}\left(c L_{x}\right), I H^{b}\left(c L_{y}\right)\right) .
\end{aligned}
$$

Now the stalk cohomology of $\operatorname{IC}^{\bullet}(X \times Y)$ is given by $H_{p}^{i}\left(\mathrm{IC}^{\bullet}(X \times Y)\right)=$ $I H^{i}(U)$, where $U$ is a basic open neighborhood in $X \times Y$ of $p=(x, y)$. Here $U$ is homeomorphic to $E \times c L$, where again $E$ is a disk of suitable dimension. In this case $c L \cong c L_{x} \times c L_{y}$, so we find the local cohomology of $\operatorname{IC}^{\bullet}(X \times Y)$ by computing the intersection cohomology groups of $c L_{x} \times c L_{y}$. The computation of $I H^{i}\left(c L_{x} \times c L_{y}\right)$ hinges on the following straightforward result:

Lemma. For topological spaces $X$ and $Y$, the product $c X \times c Y$ is homeomorphic to $c(X * Y)$.

Hence we find $H_{p}^{i}\left(\mathrm{IC}^{\bullet}(X \times Y)\right)$ using the computation of the intersection cohomology of a cone. Explicitly,

$$
H_{p}^{i}\left(\mathbf{I C}^{\bullet}(X \times Y)\right)=\left\{\begin{array}{cl}
I H^{i}\left(L_{x} * L_{y}\right), & i \leq m+n-p(m)-p(n)-2 \\
0, & i \geq m+n-p(m)-p(n)-1
\end{array}\right.
$$

where $m=\operatorname{dim}\left(c L_{x}\right)$ and $n=\operatorname{dim}\left(c L_{y}\right)$.

The link $L \cong L_{x} * L_{y}$ is contained in the complement of the union of all strata of codimension greater than $t-1$. Also notice that $\operatorname{dim}\left(L_{x}\right)<\operatorname{dim}(X)$ and $\operatorname{dim}\left(L_{y}\right)<\operatorname{dim}(Y)$, so all of the product spaces $L_{x} \times L_{y}, L_{x} \times c L_{y}$, and $c L_{x} \times L_{y}$ are of dimension less than $k$. Furthermore, if, say, $X$ is locally $\bar{p}$-torsion free (over $R$ ), then so are $L_{x}$ and $c L_{x}$. Thus, since $L$ is compact, we find $I H^{i}\left(L_{x} * L_{y}\right)$ using Proposition $3_{k-1}$, which holds by induction. The conditions in either case $(\mathrm{A})$ or $(\mathrm{B})$ then insure that

$$
H_{p}^{i}\left(\mathbf{I C}^{\bullet}(X \times Y)\right) \cong \bigoplus_{a+b=i} I H^{a}\left(c L_{x}\right) \otimes I H^{b}\left(c L_{y}\right) \oplus \bigoplus_{a+b=i-1} \operatorname{Tor}^{R}\left(I H^{a}\left(c L_{x}\right), I H^{b}\left(c L_{y}\right)\right)
$$

which completes the $(t-)$ inductive step of the proof of Proposition $2_{k}$.

\section{COUNTEREXAMPLES}

1. In this example, we show that the Künneth formula (with field coefficients) need not hold in the case where the perversity fails to satisfy the inequalities in condition (B).

Let $X=Y=c T^{2}$, and let $\bar{p}$ be a perversity such that $p(3)=0$. Check that the betti numbers for $I^{\bar{p}} H^{*}\left(c T^{2} ; \mathbb{Q}\right)$ are $(1,2,0,0)$, and that $I^{\bar{p}} H^{*}(X ; \mathbb{Q}) \otimes$ $I^{\bar{p}} H^{*}(Y ; \mathbb{Q})=(1,4,4,0,0,0,0)$. Using a Mayer-Vietoris sequence as in $\S 3$, we obtain $(1,4,4,0,0,1)$ as the betti numbers for $I^{\bar{p}} H^{*}\left(T^{2} * T^{2} ; \mathbb{Q}\right)$. 
Now $X \times Y=c T^{2} \times c T^{2} \cong c\left(T^{2} * T^{2}\right)$, so we find $I^{\bar{p}} H^{*}(X \times Y ; \mathbb{Q})$ using the computation of the intersection cohomology of a cone. For a perversity $\bar{p}$ with $p(3)=0$, there are four possible values for $p(6)$, namely $p(6)=0,1,2$, or 3 . If $0 \leq p(6) \leq 2$, then the perversity satisfies the inequalities in condition (B) and we have $I^{\bar{p}} H^{*}(X \times Y ; \mathbb{Q})=(1,4,4,0,0,0,0)=I^{\bar{p}} H^{*}(X ; \mathbb{Q}) \otimes$ $I^{\bar{p}} H^{*}(Y ; \mathbb{Q})$. However if $p(6)=3$, then $\bar{p}$ does not satisfy condition $(\mathrm{B})$ and $I^{\bar{p}} H^{*}(X \times Y ; \mathbb{Q})=(1,4,0,0,0,0,0)$.

2. This example demonstrates that the Künneth formula need not hold in the case where the perversity satisfies the inequalities in condition (B), but neither $X$ nor $Y$ is locally $\bar{p}$-torsion free.

Let $M$ denote the lens space $S^{3} /(\mathbb{Z} / n \mathbb{Z})$, and let $X=Y=c M$. If $\bar{p}$ is a perversity such that $p(4)=0$, then $X$ and $Y$ are not locally $\bar{p}$-torsion free (over $\mathbb{Z}$ ) since the link of the singular stratum (the cone point) in $c M$ is just $M$, and $I^{\bar{p}} H^{2}(M ; \mathbb{Z})=H^{2}(M ; \mathbb{Z})=\mathbb{Z} / n \mathbb{Z}$.

Now $X \times Y=c M \times c M \cong c(M * M)$, so we find $I^{\bar{p}} H^{*}(X \times Y ; \mathbb{Z})$ by again using the computation of the intersection cohomology of a cone. In particular if $p(8)=2$, then $I^{\bar{p}} H^{5}(X \times Y ; \mathbb{Z})=0$. On the other hand,

$$
\begin{aligned}
& \bigoplus_{a+b=5} I H^{a}(X) \otimes I H^{b}(Y) \oplus \bigoplus_{a+b=4} \operatorname{Tor}\left(I H^{a}(X), I H^{b}(Y)\right) \\
& =\operatorname{Tor}\left(H^{2}(M), H^{2}(M)\right)=\mathbb{Z} / n \mathbb{Z} .
\end{aligned}
$$

Notice that any perversity with $p(4)=0$ and $p(8)=2$ must satisfy condition (B).

Analogous examples involving pseudomanifolds without boundary may be obtained by considering suspensions rather than cones in each of the above.

\section{REFERENCES}

[Bo] A. Borel et al., Intersection cohomology, Birkhäuser, Boston, Mass., 1984.

[BGM] J. P. Brasselet, M. Goresky, and R. MacPherson, Piecewise linear differential forms with poles (to appear).

[Ch] J. Cheeger, On the Hodge theory of Riemannian pseudomanifolds, Geometry of the Laplace Operator, Proc. Sympos. Pure Math., vol. 36, Amer. Math. Soc., Providence, R.I., 1980.

[GM1] M. Goresky and R.MacPherson, Intersection homology theory, Topology 19 (1980), 135-162. [GM2] _, Intersection homology. II, Invent. Math. 71 (1983), 77-129.

[GS] M. Goresky and P. Siegel, Linking pairings on singular spaces, Comment. Math. Helv. 58 (1983), 96-110.

[Iv] B. Iverson, Cohomology of sheaves, Springer-Verlag, Berlin, 1986.

[Ki] H. King, Topological invariance of intersection homology without sheaves, Topology Appl. 20 (1985), 149-160.

(D. C. Cohen and M. Goresky) Department of Mathematics, Northeastern University, Boston, Massachusetts 02115

E-mail address: dcohen@northeastern.edu

E-mail address: goresky@northeastern.edu

(Lizhen Ji) Department of Mathematics, Massachusetts Institute of Technology, CamBRIDGe, MASSACHUSETTS 02139

E-mail address: ji@math.mit.edu 